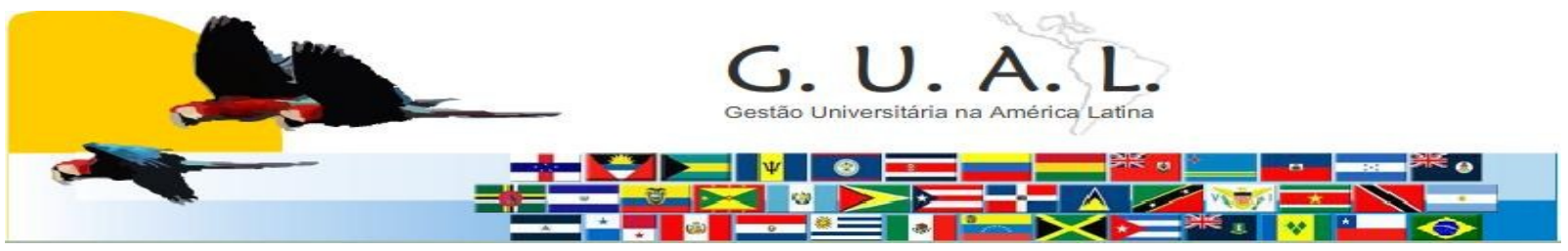

ISSN 1983-4535

\title{
UNIVERSIDADE PÚBLICA, DEMOCRÁTICA E POPULAR: OS DESAFIOS DA IMPLANTAÇÃO DA UNIVERSIDADE FEDERAL DA FRONTEIRA SUL
}

\author{
Vicente de Paula Almeida Júnior, Doutor \\ Universidade Federal da Fronteira Sul - UFFS \\ vicente.almeida@uffs.edu.br \\ Kelly Cristina Benetti Tonani Tosta, Doutora \\ Universidade Federal da Fronteira Sul - UFFS \\ kellytosta@uffs.edu.br
}

\section{RESUMO}

Este artigo tem como objetivo apresentar o processo de implantação da Universidade Federal da Fronteira Sul a partir de alguns dos condicionantes de sua implantação (traços de contexto), da concepção delineada de universidade pública, democrática, popular (conceito) e, finalmente, da constituição de sua organização institucional, multicampi e interestadual (desenho institucional). Assim, fica demonstrada a importância da consideração do entorno como peça-chave na construção das políticas e estratégias de uma instituição que nasce a partir das demandas dos movimentos sociais. Visa garantir o acesso ao ensino superior público e de qualidade, a qualificação profissional e o compromisso de inclusão social e o desenvolvimento do ensino, da pesquisa e da extensão como condição de existência de um ensino crítico, investigativo e inovador. A UFFS busca construir uma estrutura organizacional diferenciada, onde os campi são os órgãos de base e são considerados igualmente importantes, garantindo o mesmo padrão de qualidade para as condições de infraestrutura física e humana no interior de cada campus. A essência é a manutenção do diálogo constante entre as instâncias administrativas, normativas e pedagógicas, entre os órgãos superiores e de base da universidade, onde a autonomia administrativa e pedagógica acontece em todos os campi da universidade.

Palavras-chave: Gestão universitária. Estrutura multicampi. Implantação. Desenho institucional. 


\section{INTRODUÇÃO}

Este artigo tem como objeto o processo de implantação de uma universidade brasileira nova, a Universidade Federal da Fronteira Sul - UFFS. Ao tratar da peculiaridade dessa instituição, que atualmente oferta 2.160 vagas por ano em 19 tipos de cursos distribuídos em três Estados - Santa Catarina, Paraná e Rio Grande do Sul - o texto pretende contribuir com a reflexão, no âmbito das instituições federais de ensino superior - IFES, acerca das diferentes concepções de universidade multicampi implantadas ou em implantação no país, como é o caso da UFFS.

Sabemos que a reflexão sobre instituições complexas como são as universidades federais, exige considerar os múltiplos aspectos que impactam direta ou indiretamente no curso de seu desenvolvimento ou de sua trajetória. Possuindo todas as características próprias de uma organização complexa, como destaca Fischer (2001, p. 44), a universidade "com seus objetivos, estratégias, sistemas e procedimentos administrativos e técnicos, estrutura de poder e funções, entre outras", deve ser estudada fora da generalização das teorias da administração de empresas. A sua missão, objetivos e gestão seguem diretrizes próprias, inerentes à sua função científica e social na sociedade, divergindo da lógica de mercado.

Nesse trabalho apresentamos os contornos da experiência da UFFS, traçando o contexto no qual se inscreve a sua concepção, bem como o seu desenho institucional. Especificamente, apontamos alguns dos inúmeros desafios que incidem sobre o processo de implantação dessa universidade a partir (i) de alguns dos condicionantes de sua implantação (traços de contexto), (ii) da concepção delineada de universidade pública, democrática, popular (conceito) e, finalmente, (iii) da constituição de sua organização institucional, multicampi e interestadual (desenho institucional).

A figura 1, a seguir, expõe resumidamente os eixos desse raciocínio, procurando chamar a atenção para a relação presente como "pano de fundo" em toda a confecção deste texto, a complexidade de estabelecer coesão e coerência entre contexto, concepção e desenho institucional no processo de implantação de uma universidade nova, multicampi e interestadual. 


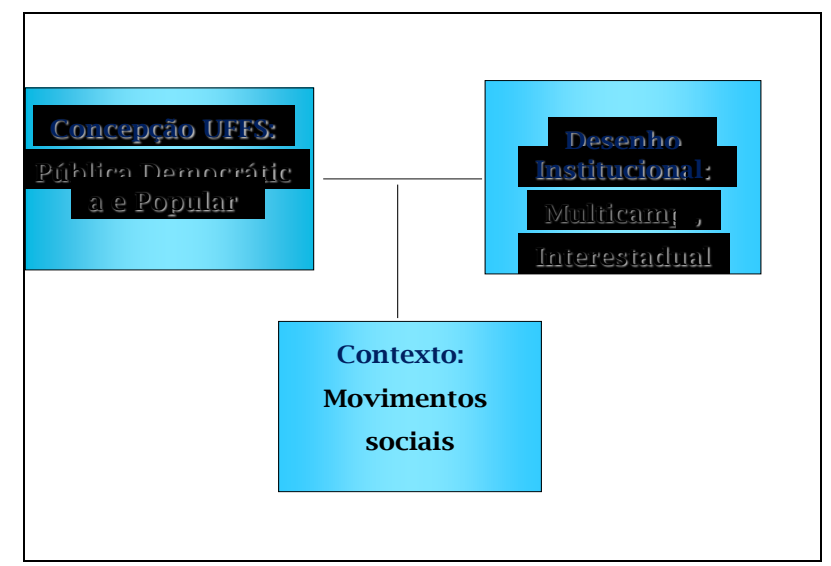

Figura 1 Contexto, Concepção e Estrutura da UFFS

Fonte: Elaborada pelos autores

A fim de alcançar o nosso propósito, o texto está organizado em duas seções. Na primeira, descrevemos os traços de contexto no qual se delineou a concepção da UFFS. A UFFS nasce a partir de demandas dos movimentos sociais organizados a partir de 2005 no Movimento Pró-Universidade Federal. Criada oficialmente em 15 de setembro de 2009, pela Lei $\mathrm{n}^{\mathrm{o}}$ 12.029, a universidade inicia suas atividades letivas em março de 2010. Observamos que a sua existência tem coincidência com as demandas dos movimentos sociais por acesso à educação superior pública, abarcando aproximadamente 396 municípios da mesorregião da Fronteira do MERCOSUL, desde o sudoeste do Paraná, passando pelo oeste de Santa Catarina, até o noroeste do Rio Grande do Sul, historicamente excluídos do processo de desenvolvimento percebido nas áreas mais próximas do litoral brasileiro.

Na segunda seção, apresentaremos o atual desenho institucional da UFFS. Consoante com a sua Lei de criação e com o seu Estatuto, a universidade já nasce como instituição multicampi e interestadual, com a missão de conferir unicidade acadêmica, pedagógica, administrativa em todos os seus cinco campi, diferentemente de algumas universidades federais que, somente após a consolidação de sua existência, expandiram de forma multicampi.

\section{TRAÇOS DE CONTEXTO DA UFFS: HISTÓRICO DE IMPLANTAÇÃO E CONCEPÇÃO DE UNIVERSIDADE PÚBLICA, DEMOCRÁTICA E POPULAR}

Esta seção apresenta, de forma sucinta, o caminho percorrido pela Universidade Federal da Fronteira Sul - UFFS e a concepção de universidade construída ao longo de seus dois anos de existência. Muitas das informações aqui descritas têm como referência o 
Documento que sistematizou um importante evento organizado na fase inicial de implantação da UFFS - I Conferência de Ensino, Pesquisa e Extensão da UFFS (COEPE) realizada entre junho a setembro de 2010. Esse documento, publicado em 2011, intitula-se: Construindo agendas e definindo rumos: I Conferência de Ensino, Pesquisa e Extensão da UFFS.

Desde a sua criação, a UFFS desenhou três grandes objetivos, quais sejam: (i) promover o desenvolvimento regional integrado (condição essencial para a garantia da permanência dos cidadãos na região da fronteira sul e a reversão do processo de litoralização hoje em curso); (ii) assegurar o acesso ao ensino superior como fator decisivo para o desenvolvimento das capacidades econômicas e sociais da região, a qualificação profissional e o compromisso de inclusão social e (iii) desenvolver o ensino, a pesquisa e a extensão como condição de existência de um ensino crítico, investigativo e inovador e a interação entre as cidades e estados que compõem a grande fronteira do Mercosul e seu entorno.

Sabe-se que a Mesorregião da Fronteira Sul, onde se situa a UFFS, tem a agropecuária e a agroindústria como base de sua estrutura produtiva e um grau de urbanização relativamente baixo em relação ao restante do País, concentrando parcela significativa da população na zona rural.

Em relação à região Sul, a Mesorregião representa um quarto do território e da população. No entanto, o PIB da Mesorregião representa pouco mais de um décimo e o PIB per capita é $40 \%$ menor que o da região Sul. Além disso, os indicadores demonstram que a Mesorregião, em relação à Região Sul e ao Brasil, vem empobrecendo.

Essa realidade induziu o deslocamento populacional do campo para a cidade, dos pequenos municípios para as cidades-pólo de cada microrregião e, principalmente, da Mesorregião para outras regiões, num processo conhecido como litoralização da população.

Tal movimento vem minando as forças produtivas locais e dificultando a geração de um processo de desenvolvimento endógeno. Essa condição na região, somado ao fato de estar ocupada basicamente por instituições privadas de ensino, condicionaram à sua população a cobrança de mensalidades para cursar o nível superior, excluindo os mais carentes. Assim, a exclusão tem um claro recorte social, visto que impede justamente o acesso ao ensino superior da população com menor poder aquisitivo. Esse processo, além de excludente contribui para extrair renda da população mais pobre. $\mathrm{Na}$ área da pesquisa e da extensão, os limites são ainda maiores.

Rev Rev. GUAL., Florianópolis, Edição especial 2011, p.25-36. 
Por essa razão, a oferta de ensino superior público e gratuito, especialmente à população mais carente, articulada com um forte investimento em pesquisa e extensão, que atenda os três Estados da Mesorregião com diversos campi, é condição essencial ao desenvolvimento regional. É nesse contexto que se inseriu a ação $125 \mathrm{C}$ do Ministério da Educação (MEC) - Implantação da Universidade Federal da Fronteira Sul - como estratégia para a promoção do desenvolvimento regional.

Para alcançar sua missão e seus objetivos, a Universidade Federal da Fronteira Sul se constituiu a partir de uma estrutura multicampi. A proposição de localização da sede e dos campi da Universidade tomou como base os seguintes critérios: regiões com forte presença de organizações e Movimentos Sociais Populares e do Movimento Pró-Universidade Federal; regiões com maior número de alunos no Ensino Médio; regiões com IDH mais baixos; regiões que representem centralidade no contexto geográfico da Mesorregião e que contenham certa infra-estrutura de transporte, comunicação, educação básica, servidores públicos, hotéis; distribuição dos campi entre os estados, considerando o número de municípios e a população da Mesorregião e seu entorno. O quadro 1, UFFS em Números 2010, apresenta alguns dados relativos ao primeiro ano de existência:

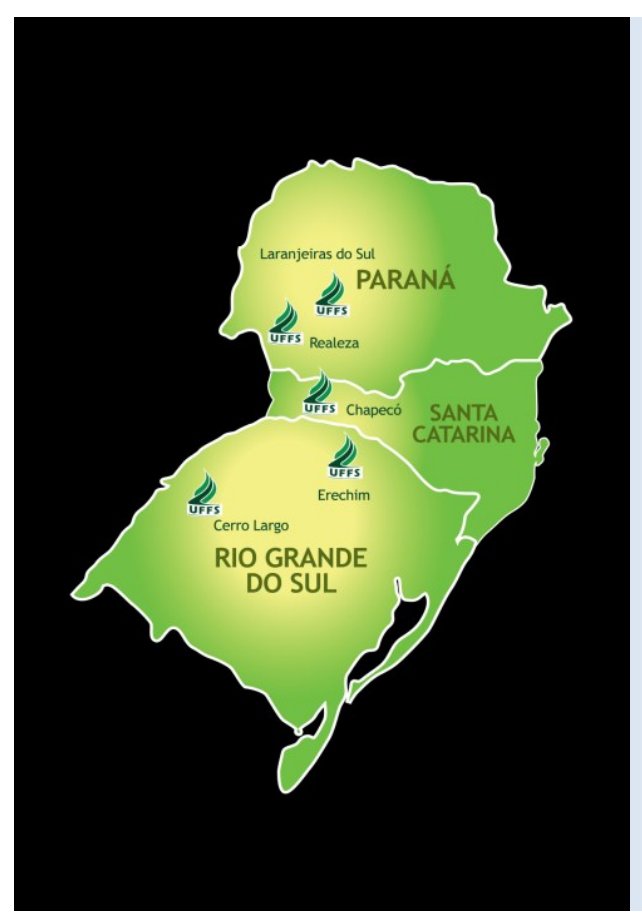

\section{UFFS em Números}

05 Campi

3979 Alunos ativos e trancados

288 Professores efetivos

102 Doutores

189 Mestres

275 Técnicos-administrativos

N IVEL MÉDIO 56

GRADUAÇÃO 136

ESPEC IALIZAÇAO 71

MESTRADO 12

42 ofertas

33 cursos cadasatrados e-mec

19 cursos diferentes

Quadro 1 UFFS em Números 2010.

Fonte: Diretoria de Comunicação UFFS, 2010

Rev Rev. GUAL., Florianópolis, Edição especial 2011, p.25-36. 
Nesse quadro, a UFFS vem se desenvolvendo para consolidar-se enquanto universidade pública e popular, comprometida com a formação técnica e ética dos cidadãos e com o desenvolvimento sustentável e solidário da Região Sul do país; Universidade democrática, autônoma, que respeita a pluralidade de pensamento e diversidade cultural, que garante os espaços de participação dos diferentes sujeitos sociais; Universidade que estabeleça dispositivos de combate às desigualdades sociais e regionais, incluindo condições de acesso e permanência no ensino superior, especialmente da população mais excluída do campo e da cidade, Universidade que tenha na agricultura familiar um setor estruturador e dinamizador do processo de desenvolvimento e que tenha como premissa a valorização e a superação da matriz produtiva existente (UFFS, 2011, p. 31-34).

Assim, com base nessas características, a concepção de universidade assumida pela UFFS visa garantir em essência (i) o acesso ao ensino superior público e de qualidade, (ii) a qualificação profissional e o compromisso de inclusão social e (iii) o desenvolvimento do ensino, da pesquisa e da extensão como condição de existência de um ensino crítico, investigativo e inovador.

\subsection{O desenho institucional: algumas características e questões sobre a estrutura organizacional da UFFS}

A UFFS, de acordo com o seu Estatuto aprovado em 21 de setembro de 2010, apresenta em sua estrutura órgãos superiores e órgãos de base.

Os órgãos superiores são de Administração (Reitoria), de Deliberação (Conselho Universitário - CONSUNI), de Supervisão e Fiscalização (Conselho Curador, Auditoria Interna) e Consultivo (Conselho Estratégico Social).

O Estatuto da UFFS define como órgãos de base os cinco campi da UFFS: Campus Chapecó, em Santa Catarina (Campus sede), Campus Realeza e Campus Laranjeiras do Sul, no Paraná, e Campus Erechim e Campus Cerro Largo, no Rio Grande do Sul. Pertencem à base os órgãos de Administração (Direção do Campus e Coordenação de Cursos) e órgãos de Deliberação (Conselho de Campus e Colegiado de Cursos). O órgão Consultivo, presente nos campi é o Conselho Comunitário de Campus. Abaixo, o quadro 2 - Organograma da UFFS mostra a atual arquitetura institucional da universidade. 


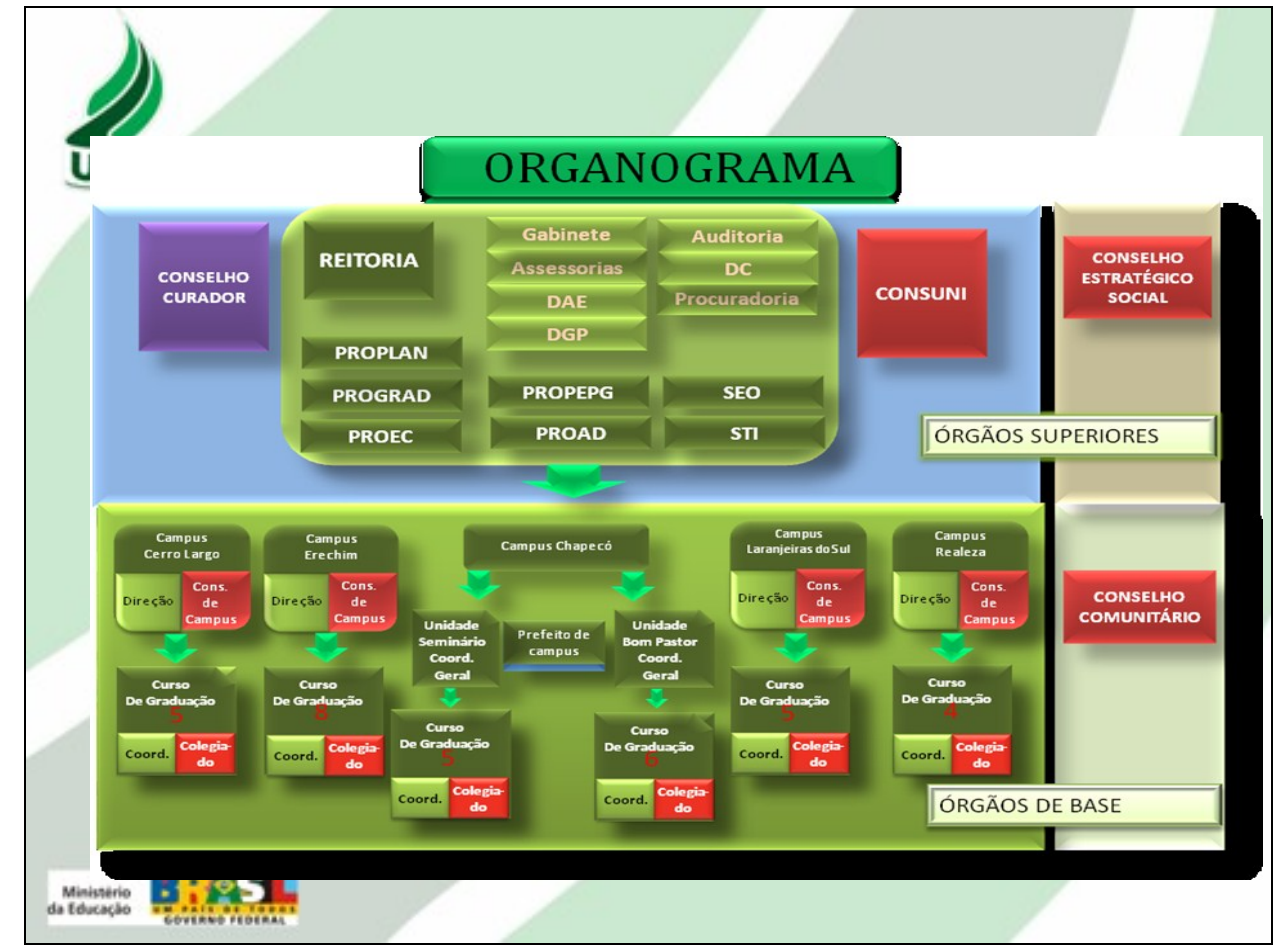

Quadro 2 Organograma da UFFS.

Fonte: PROPLAN - UFFS, 2011.

Observa-se que, conforme ilustra o Quadro 2, existe uma relação dialógica entre as estruturas superiores e as de base na universidade. Ou seja, os órgãos administrativos e deliberativos constantes para garantir o bom funcionamento e desenvolvimento institucional da UFFS estão presentes igualmente na sua base, os campi. Isso representa o esforço para conferir diálogo, representatividade e condições de gestão equitativas em todos os campi da universidade.

Nessa perspectiva, garantida pela Lei de Criação da UFFS e pelo seu Estatuto, todos os campi têm a mesma importância para a construção das Políticas voltadas ao Ensino, Pesquisa, Extensão e Cultura, assim como para o funcionamento e consolidação do processo de implantação da universidade. Como é uma instituição multicampi, todo o seu desenho, formado pelos órgãos superiores e de base, orientam todas as peças normativas e as ações relacionadas à gestão da universidade.

Existe, porém, alguns pontos que merecem atenção em relação à realidade desse desenho. Pelo fato de não significar um mero conjunto ou reunião de campi instalados em três Estados do país (Santa Catarina, Paraná e Rio Grande do Sul), o Estatuto da UFFS prevê, como um dos objetivos para a consolidação da Universidade, Multicampi e Interestadual, a 
articulação orgânica de sua base, isto é, de seus campi. Tal articulação colide, hoje, com a tendência histórica da segmentação, separação, departamentalização presentes, ainda, em boa parte das universidades brasileiras.

Outro ponto, igualmente relevante, tem relação com a o desafio de garantir padrão de qualidade para as condições de infraestrutura física e humana no interior de cada campus. Num desenho multicampista, isso é fundamental para promover identidade e sinergia na concretização da missão e dos objetivos institucionais. No caso da UFFS, por exemplo, todos os campi caminham, pois estão em construção, para o mesmo padrão e estrutura física, possuindo biblioteca universitária, blocos de salas de aula, bloco para abrigar as salas dos professores, espaço de convivência social e cultural, restaurante universitário, entre outras instalações.

Portanto, a configuração socioespacial multicampi da UFFS, a institucionalização das dimensões normativas na universidade, estruturais e culturais não ocorre apenas na sede, mas em todos os campi a partir de uma relação dialógica entre as instâncias administrativas, normativas e pedagógicas, entre os órgãos superiores e de base da universidade.

A questão da autonomia administrativa e pedagógica, prerrogativa estatutária da Administração Central, acontece também nos campi da universidade. Embora os campi têm participação e atendem as diretrizes gerais da Universidade, integrando diariamente as linhas e políticas de trabalho consolidadas por meio das Pró-reitorias, seguem produzindo e gerenciando os processos de forma mais eficaz de acordo com a realidade local e regionalmente.

No que diz respeito à autonomia orçamentária e financeira, a UFFS, como instituição nova e em fase de implantação, não participa da matriz de distribuição proposto pela Associação Nacional de Dirigentes das Instituições Federais de Ensino Superior - ANDIFES. Seu orçamento e recursos são, respectivamente, frutos de um processo pactuado com a Secretaria de Orçamento Federal (SOF) e o Ministério da Educação (MEC) e estavam atrelados até o ano de 2010 ao orçamento de sua tutora, a Universidade Federal de Santa Catarina - UFSC.

Somente a partir de 2011 foi criado uma Unidade Orçamentária (UO 26440) para a toda a UFFS. Desde então, a UFFS vem executando o seu próprio orçamento, mediante o empenho, liquidação e pagamento das despesas relacionadas à implantação física e despesas 
ligadas ao seu funcionamento para garantir o pleno desenvolvimento dos cursos de graduação, projetos e atividades de extensão, pesquisa e pós-graduação lato-sensu.

No contexto interno da instituição, tais recursos, repassados pelo MEC, são alocados conforme descentralização temática, isto é, por meio de ações orçamentárias (Implantação da UFFS, Funcionamento dos Cursos de Graduação e Pós-graduação, Fomentos às Ações de Ensino, Pesquisa e Extensão, Capacitação de Servidores em Processo de Qualificação e Requalificação, Assistências aos Estudantes, entre outras ações) para o conjunto da UO UFFS e não, conforme poderia se supor, alocados por setor ou campus.

Esse aspecto é bastante interessante porque confere, a nosso ver, maior possibilidade de planejamento transversal e desenvolvimento institucional de modo equânime entre os campi, evitando disputas muitas vezes corporativas pelo poder e por maiores recursos. Em resumo, não se estabelece a priori a relevância de determinado campus em relação ao outro, o que evita a possibilidade de campus desenvolvido e outro menos desenvolvido numa mesma universidade.

A questão da Comunicação Institucional representa um dos maiores desafios numa universidade multicampi. Tendo em vista que os campi distam geograficamente entre si, o acesso às informações relevantes para a execução das atividades depende diretamente de um modelo desconcentrado da Administração Central. Quando não existe um sistema cooperativo e circular na instituição, baseado nas mais recentes tecnologias da informação, a tendência é gerar insegurança nos gestores, distorções sobre os acontecimentos da vida universitária e insatisfação na comunidade de estudantes e professores.

A UFFS minimiza esse desafio buscando conciliar o agendamento constante de reuniões centralizadas e descentralizadas, presenciais e à distância, combinadas por meio de múltiplos instrumentos de informação, como murais impresso e eletrônico, sítio atualizado pelo próprio setor, agenda comum da UFFS entre outras formas. Mas isso, na prática, pode se revelar ainda mais desafiador quando os atores que formam a instituição denominada de Universidade têm dificuldade de compreender claramente a missão e os objetivos dela, quando têm dificuldade de organizar as ações, atividades, projetos, planos e programas para um mesmo fim.

Rev Rev. GUAL., Florianópolis, Edição especial 2011, p.25-36. 


\section{CONSIDERAÇÕES FINAIS}

Como qualquer sistema aberto e, principalmente pelos objetivos que fundamentam sua existência, a Universidade interage acentuadamente com o meio onde está inserida, influenciando e sendo influenciada por ele. A UFFS aproveita essa interação na construção diária de uma instituição pública, democrática e popular

Nota-se que a legislação já supõe que a universidade não existe de maneira isolada do ambiente onde está inserida, uma vez que além do ensino, esta necessariamente terá de fazer pesquisa, ou seja, produzir novos conhecimentos e fazer extensão, que é aplicar e difundir esse conhecimento com a sociedade. Infelizmente essa não é a prática que vemos refletida na imagem das instituições públicas federais brasileiras.

É necessário que os gestores atuem de modo que o papel da Universidade vá além da formação de profissionais e a transforme em uma instituição com objetivos mais amplos que a transmissão do conhecimento.

O entorno exerce papel importante na construção das políticas institucionais da UFFS, principalmente porque ela é a primeira instituição brasileira que nasce "de baixo para cima". Sua atual estrutura organizacional reforça essa intenção quando institucionaliza o papel opinativo da sociedade na condição de conselho consultivo.

“A implementação desse novo modelo de universidade mais interativa permite às instituições entrarem na contemporaneidade, alinhadas com os caminhos de $\mathrm{u}$ m mundo sem fronteiras, da era do conhecimento virtual e idéias compartilhadas" (MELO, 2008, p. 239).

A UFFS vem sendo pensada como uma instituição com um modelo inovador, efetivamente integrado entre si, ou seja, entre seus cinco campi, e com o meio onde está inserida e de onde nasceu.

Ao considerar o campus como principal órgão de base, a UFFS reforça a representatividade dos campi onde a Universidade acontece de fato, e o constante diálogo entre esses órgãos e a Administração e demais órgãos superiores garante a unicidade de política e de estratégia, que a difere das instituições tradicionais. 


\section{REFERÊNCIAS}

FISCHER, Augusto. Mudança organizacional na universidade: o caso da UNOESC Campus de Videira-SC. 2001. 147 f. Dissertação (Mestrado em Engenharia de Produção) Programa de Pós-Graduação em Engenharia de Produção. Universidade Federal de Santa Catarina, Florianópolis, 2001.

MELO, Pedro Antônio de. A autonomia universitária e seus reflexos na gestão e nos resultados de Universidades brasileiras. In: LANER, Aline dos Santos.; CRUZ JUNIOR, João Benjamim da. Indivíduo, organizações e sociedade. Ijuí: Unijuí, 2008. p. 245-260.

UFFS - UNIVERSIDADE FEDERAL DA FRONTEIRA SUL. Construindo agendas e definindo rumos: I Conferência de Ensino, Pesquisa e Extensão da UFFS. TREVISOL, J. V., CORDEIRO, M. H., HASS, M. (orgs.). Chapecó, SC: UFFS, 2011.

UFFS - UNIVERSIDADE FEDERAL DA FRONTEIRA SUL. Estatuto. Chapecó, SC: UFFS, 2010.

UFFS - UNIVERSIDADE FEDERAL DA FRONTEIRA SUL - Diretoria de Comunicação. UFFS em números. Chapecó, SC: UFFS, 2010. 


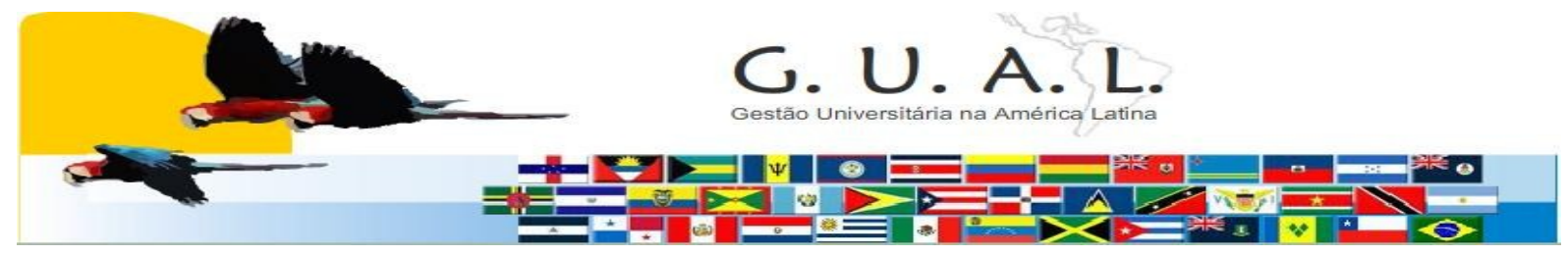

ISSN 1983-4535

\title{
PUBLIC, DEMOCRATIC AND POPULAR UNIVERSITY: THE CHALLENGES OF IMPLEMENTATION OF THE UNIVERSIDADE FEDERAL DA FRONTEIRA SUL
}

\author{
Vicente de Paula Almeida Júnior, Doctor \\ Universidade Federal da Fronteira Sul - UFFS \\ vicente.almeida@uffs.edu.br \\ Kelly Cristina Benetti Tonani Tosta, Doctor \\ Universidade Federal da Fronteira Sul - UFFS \\ kellytosta@uffs.edu.br
}

\begin{abstract}
This article aims to present the process of implementation of the Universidade Federal da Fronteira Sul from some of the conditions of its implementation (context lines), the outlined concept of a public, democratic and popular university (concept) and, finally, the constitution of its institutional organization, interstate and multicampi (institutional design). Thus, it demonstrates the importance of considering the environment as a key part in building policies and strategies of an institution that is born from the demands of the social movements. It aims to ensure access to public and quality higher education, professional qualifying and commitment to social inclusion and development of education, research and extension as a condition of existence of a critical, researcher and innovator teaching. UFFS seeks to build a different organizational structure, where the campi are the basic organs and are considered equally important, ensuring the same quality standard for the conditions of physical and human infrastructure within each campus. The essence is to maintain constant dialogue between the administrative, regulatory and educational instances, among the superior and basic organs of the university, where the administrative and pedagogical autonomy takes place in all university campi.
\end{abstract}

Keywords: University management. Structure multicampi. Implementation. Institutional design. 